\title{
Effect of CNT Diameter on Physical Properties of Styrene-Butadiene Rubber Nanocomposites
}

\author{
Young Soo Park ${ }^{1,3}$, Mongyoung Huh ${ }^{1,3}$, Sin Jae Kang²,3, Seok Il Yun ${ }^{3}$ and Kay Hyeok Ahn, \\ ${ }^{1}$ Department of Polymer-nano Science and Technology, Chonbuk National University, Jeonju \\ ${ }^{2}$ Department of Mechanical Design Engineering, Chonbuk National University, Jeonju, 561-756, Korea \\ ${ }^{3}$ Nano Material Department, Jeonju Institute of Machinery and Carbon Composites, Jeonju, 644-841, Korea \\ •e-mail: khan@jmrc.re.kr \\ (Received November 5, 2009; Accepted December 18, 2009)
}

\begin{abstract}
We investigated the effect of diameter and content of carbon nanotubes (CNTs) on the physical properties of styrenebutadiene rubber (SBR)/CNTs nanocomposites. CNTs-reinforced SBR nanocomposites were prepared by the melt mixing process. CNTs with different diameters were synthesized by the chemical vapor deposition method (CVD). In this work, the mechanical property and other physical properties of SBR/CNTS nanocomposites were discussed as a function of the content and diameter of CNTs.
\end{abstract}

Keywords : Carbon nanotube, Styrene-butadiene rubber, Diameter, Mechanical properties

\section{Introduction}

The styrene-butadiene rubber (SBR) is one of the most widely used rubbers due to its inexpensive cost and good mechanical property. However SBR lacks the selfreinforcing qualities of natural rubber due to the stress induced crystallization, gum vulcanization of SBR leading to lower mechanical properties. The CNTs have drawn considerable interests as reinforcing materials [1-5], since CNTs have high values of strength and modulus, which are as almost $200 \mathrm{GPa}$ and $1 \mathrm{TPa}$, respectively [6-8]. The diameter of CNTs has significant effects on their mechanical properties. The mechanical strength of CNTs was found to decrease from 1 to $0.1 \mathrm{TPa}$, as the CNT diameter increased from 8 to $40 \mathrm{~nm}$ [9]. The mechanical properties of CNTs/ rubber nanocomposites have been previously reported [10-14]. However the relationship between mechanical properties of the nanocomposites and the diameter of CNTs has not been studied.

The purpose of this work was to evaluate the influence of the CNT diameter on the mechanical behavior and cure property of CNT/SBR nanocomposites.

\section{Experimental}

\subsection{Materials}

A styrene-butadiene rubber, SBR (Kosyn 1500, styrene content: $23.5 \%$, Mooney Viscosity ML1+4 at $100^{\circ} \mathrm{C}$ : 52 , specific gravity: 0.93) was provided by Kumho Petrochemical. Zinc oxide and stearic acid were used as an activator, and N-tert-butyl-2-benzothiazole-sulfenamide (NS, Flexsys, USA) and sulfur were selected for the curing system.

CNTs were prepared by the following process. Three different components of co-catalyst Fe-Mo in $\mathrm{MgO}$ support were prepared by the impregnating method. The synthesis of CNTs was carried out in the CVD reactor with the flow of methane and hydrogen gases at $900^{\circ} \mathrm{C}$.

\subsection{Preparing and characterization of purified CNTS}

Synthesized CNT samples had different amount of CNTs. To decrease the effects of $\mathrm{MgO}$ and Mo-Fe on the property of nanocomposites, CNT samples were first ground with mixer and treated with a mild $\mathrm{HNO}_{3}$ solution for $2 \mathrm{hr}$. Herewith, the nanotubes were washed with deionized water until the $\mathrm{pH}$ of the washings was around 7. Finally the nanotubes were filtered with PTFE filter and dried in $120^{\circ} \mathrm{C}$ for $24 \mathrm{hr}$. The synthesized and purified CNTs were analyzed for morphologies of CNT powders by SEM (Scanning Electron Microscope), for the averages and distributions of diameters by TEM (Transmission Electron Microscope), for the crystalline of CNTs by Raman spectroscope, and for the purity of CNT powders by TGA (Thermo-Gravimetric Analysis).

\subsection{Preparation of CNT/SBR nanocomposites}

CNTs/Rubber compounds were prepared in an internal 
Table 1. Charaterization of CNTs

\begin{tabular}{cccc}
\hline $\begin{array}{c}\text { Product } \\
\text { name }\end{array}$ & Property & Value & $\begin{array}{c}\text { Method of } \\
\text { measurements }\end{array}$ \\
\hline \multirow{2}{*}{ CNT-1 } & diameter & $1 \sim 4 \mathrm{~nm}$ & TEM \\
& average diameter & $1.9 \mathrm{~nm}$ & TEM \\
& IG/ID & 15 & Raman \\
& Impurity & $9 \mathrm{wt.} \%$ & TGA \\
\hline CNT-2 & diameter & $4 \sim 7 \mathrm{~nm}$ & TEM \\
& average diameter & $5.2 \mathrm{~nm}$ & TEM \\
& IG/ID & 8 & Raman \\
& Impurity & $7 \mathrm{wt} . \%$ & TGA \\
\hline \multirow{2}{*}{ CNT-3 } & diameter & $8 \sim 12 \mathrm{~nm}$ & TEM \\
& average diameter & $9.8 \mathrm{~nm}$ & TEM \\
& I G $/$ ID & $>5$ & Raman \\
\hline
\end{tabular}

mixer (Haake Rheocord 9000, Germany) for $10 \mathrm{~min}$ at a rotating speed of $60 \mathrm{rpm}$ and dumped at about $150^{\circ} \mathrm{C}$. The curing chemicals were incorporated into the nanocomposites at room temperature. The curing package was given in Table 1. The total compounding cycle was finished within $30 \mathrm{~min}$. The curatives were then mixed in a two-roll mill(8422, Farrel Co. USA) according to the procedure described in ASTM D3182 and D3184. The friction ratio of the rolling mill was 1:1.2 during the mixing tenure.

\subsection{Measurement of curing characteristics and mechani- cal properties of nanocomposites}

After the mixing process the stocks were cured under pressure of $20 \mathrm{MPa}$ in a heated press (Carver wabash, USA) at $160^{\circ} \mathrm{C}$ to the optimum cure in respect to the 190 vulcanization time determined with an oscillating disk rheometer (ODR -2000, Alpha Technologies, USA). Tensile tests have been done with a material testing machine (United STM-10, USA) with a crosshead speed of $500 \mathrm{~mm} / \mathrm{min}$.

\section{Results and Discussion}

The TEM was used to measure the distribution and average diameters of CNTs. The diameter distributions of CNTs-1, CNT-2, and CNT-3 turned out to be around $1 \sim 4 \mathrm{~nm}, 3 \sim 7 \mathrm{~nm}$, and $8 \sim 12 \mathrm{~nm}$, and the average diameter was $1.9,5.2$, and $9.8 \mathrm{~nm}$, respectively. The TEM images in left side of Fig. 1 represented for diameters of CNTs in each samples. From SEM images, it was found that that CNTs in all samples were entangled severely and especially CNTs in (a) were composed of bundles. The Table 1 shows the characterization of CNTs samples. The values of the $\mathrm{I}_{\mathrm{G}} / \mathrm{I}_{\mathrm{D}}$ determined by Raman spectroscopy were 12, 8, and 5 from CNT-1 to CNT-3 respectively which is higher than those for CNTs-rubber nanocomposites studied elsewhere [10-14].
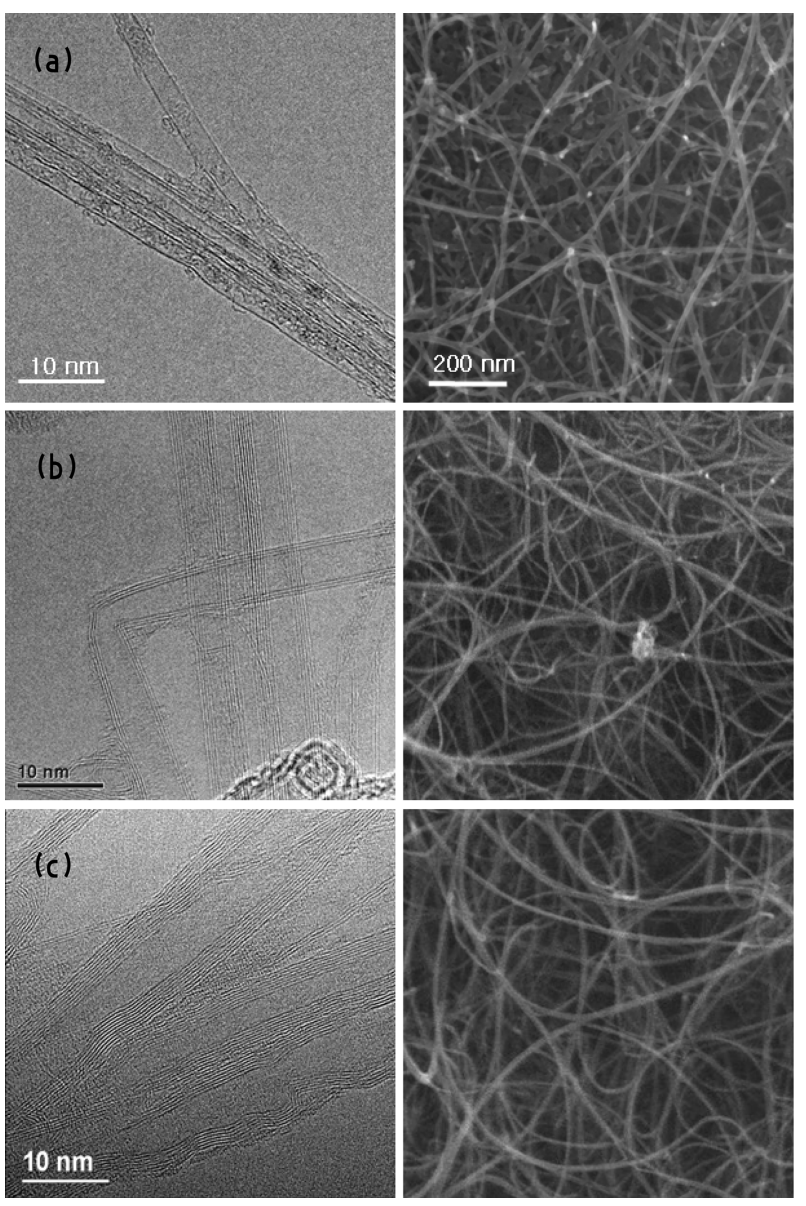

Fig. 1. Images of SEM and TEM for CNTs samples (a) CNT-1, (b) CNT-2, and (c) CNT-3.

Table 1 showed TGA data of three as-grown samples (CNT content, 40, 75 and $90 \mathrm{wt} \%$ ). According to TGA data, $\mathrm{MgO}$ were present in all samples. It was well-known that $\mathrm{MgO}$ was used as an activator during the rubber compound process. In order to investigate the CNT effect alone, $\mathrm{MgO}$ should be removed from the samples. The removal of $\mathrm{MgO}$ was performed by mild acidic treatment. After the mild acidic treatment, purities of CNT-1, CNT-2 and CNT-3 were about 91, 93 and 96 wt each (Table 1).

Fig. 2 is SEM images of rubber nanocomposites with different CNT loadings ( 3 and 5 phr of CNT). For 1 phr of CNTs content, we couldn't find any CNTs in nanocomposites with all three CNTs (CNT-1, -2, -3). The CNTs were shown in (a), (c) and (e) in Fig. 2 and indicated with arrows for each CNTs. With increasing the diameter of CNTs as from CNT-1 to CNT-3, more CNTs were shown in the same area of images and all of them appeared to be cut and surrounded by rubber. In case of CNT content, 5phr as shown in Fig. 2 (b,d,f), much more CNTs were observed compared to the nanocomposites with CNT content 3 phr. The CNTs shown in Fig. 2 (b,d,f) appeared to be pulled out from rubber composites, the 

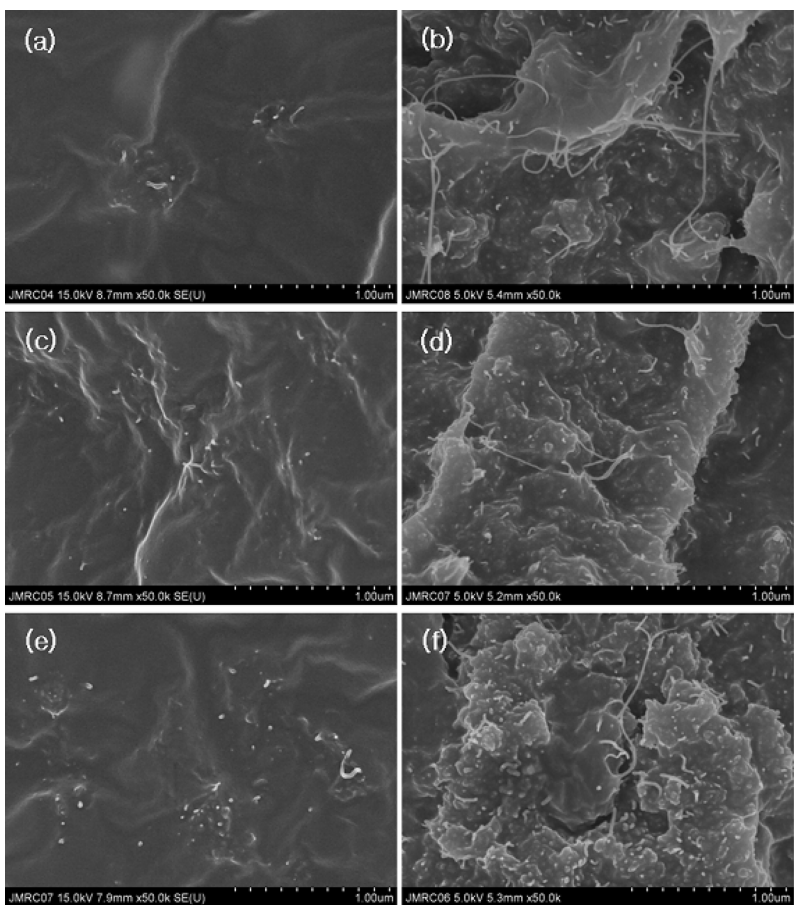

Fig. 2. Images of SEM for CNTs/rubber nanocomposites with $3 \operatorname{phr}(\mathrm{a}, \mathrm{c}, \mathrm{e})$ and $5 \operatorname{phr}(\mathrm{b}, \mathrm{d}, \mathrm{f})$ of CNT-1(a, b), CNT-2(c, d) and CNT-3(e, f).

number of pulled CNTs were decreased from (b) to (f). It was thought that the bonding between CNTs and rubber molecules became stronger with the increase of CNT diameters.

The vulcanizing kinetic parameters of rubber materials could be obtained conveniently using the vulcanizing curve, which reflected curing degrees with time at a constant temperature [14]. In this study, we investigated the vulcanizing curves of the rubber compounds at $160^{\circ} \mathrm{C}$. The vulcanization properties obtained from the vulcanization curves (Fig. 3) were shown in Table 3. Three regions such as minimum torque, curing reaction, maximum torque region, could be shown clearly in the vulcanizing curves for a typical accelerated sulfur vulcanization process [15]. In the minimum torque region, the value of torque reflected viscosity of the compound. Regardless of the diameters of CNTs, all the values of minimum torque was increased with the CNT addition as similar to previous results [10,14]. And there was the difference in the degree of increase of torque value. During the curing reaction region, the cross-linked networks between rubber molecules were formed and gradually increased with time. This appeared to increase torque value and stiffness of rubber nanocomposite. In the maximum torque, the cross-linked network in rubber is almost mature. So there was no change of torque value and viscosity of a nanocomposite. All the maximum torques of rubber nanocomposites increased with both loadings of
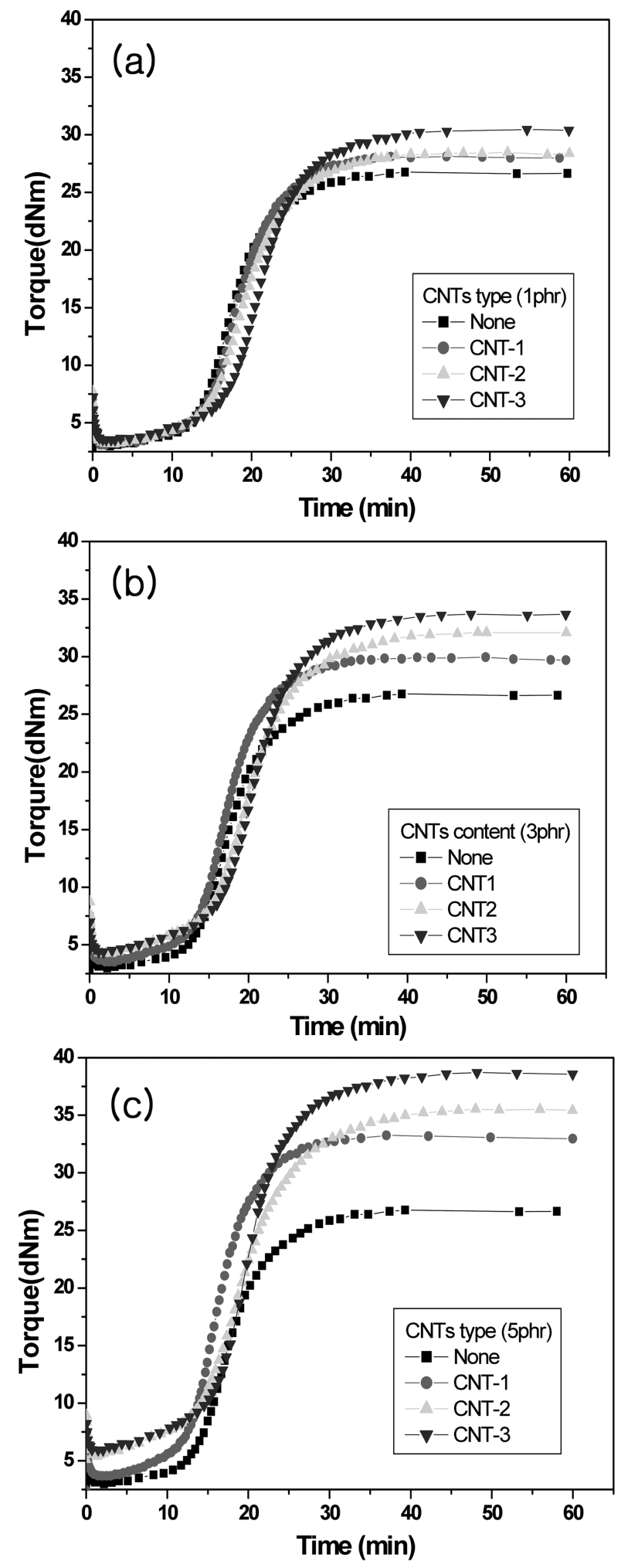

Fig. 3. Vulcanizing curves of $\mathrm{CNTs} / \mathrm{SBR}$ nanocomposites with different loading of CNTs ; (a) $1 \mathrm{phr}$, (b) $3 \mathrm{phr}$ and (c) 5 phr.

CNTs as almost same as other's works $[10,14]$. And there 
Table 2. Compound recipes investigated

\begin{tabular}{cc}
\hline Ingredient & phr \\
\hline $\mathrm{SBR}^{\mathrm{a})}$ & 100 \\
$\mathrm{CNT}^{\mathrm{b})}$ & $0,1,3,5$ \\
$\mathrm{ZnO}$ & 5 \\
Stearic acid & 2 \\
$\mathrm{NS}^{\mathrm{c})}$ & 1 \\
Sulfur & 2 \\
\hline
\end{tabular}

a) SBR1500: styrene-butadiene rubber with $23.5 \%$ of styrene content b) CNTs: CNT-1, CNT-2, and CNT-3 (purified)

c) NS: N-tert-butyl-2-benzothiazolesulfenamide

Table 3. Charaterization of CNTs

\begin{tabular}{ccccccccccccc}
\hline $\begin{array}{c}\text { Product } \\
\text { Name }\end{array}$ & None & \multicolumn{1}{c}{ CNT-1 } & \multicolumn{3}{c}{ CNT-2 } & \multicolumn{3}{c}{ CNT-3 } \\
\hline $\begin{array}{c}\text { Content } \\
\text { (phr) }\end{array}$ & 0 & 1 & 3 & 5 & 1 & 3 & 5 & 1 & 3 & 5 \\
t90(min) & 26.0 & 26.7 & 24.8 & 23.1 & 28.8 & 29.4 & 29.6 & 29.4 & 29.3 & 27.9 \\
$\begin{array}{c}\text { Min. Torque } \\
\text { (N.m) }\end{array}$ & 3.1 & 3.2 & 3.6 & 3.8 & 3.3 & 4.4 & 5.7 & 3.6 & 4.6 & 6.0 \\
$\begin{array}{c}\text { Max.Torque } \\
(\mathrm{N} \cdot \mathrm{m})\end{array}$ & 27.2 & 28.7 & 30.5 & 33.5 & 29.5 & 32.3 & 36.1 & 30.9 & 34.2 & 39.1 \\
$\Delta \mathrm{S}$ & 24.1 & 25.5 & 26.9 & 29.7 & 26.2 & 27.9 & 30.4 & 27.2 & 29.7 & 33.1 \\
\hline
\end{tabular}

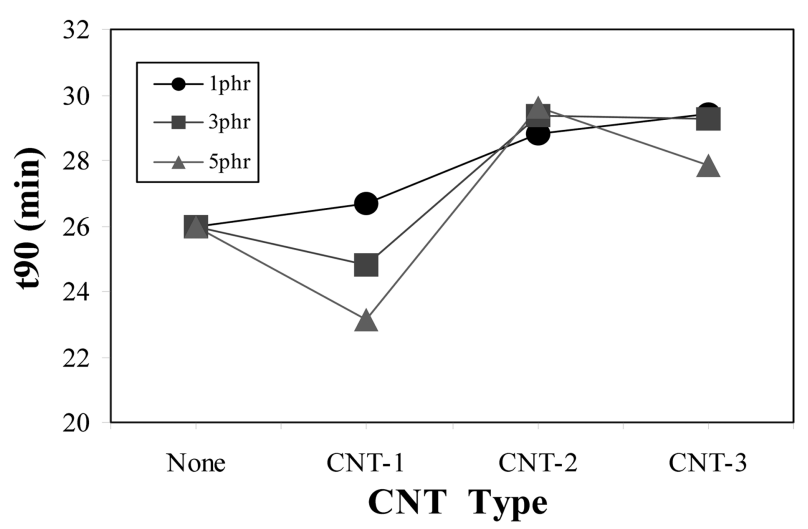

Fig. 4. Cure times with the CNT contents and diameters.

was the difference in the degree of increase of torque value as similar to minimum torques.

Variations of delta torque with the diameters and loadings of CNTs were plotted in Fig. 4. The delta torque $(\Delta \mathrm{S})$ was the difference between the maximum torque and minimum torque. The delta torque was correlated closely with the crosslink density. The delta torque was found to slightly increase with the increase of diameters and contents of CNTs. In general CNTs with a larger diameter are easily cut leading to better distribution of CNTS within the rubber matrix, which might be responsible for increased torque.

Fig. 4 showed variations of the cure time (t90) with the CNT contents and diameters. In case of nanocomposites with CNT-2, the curing time slightly increased with the

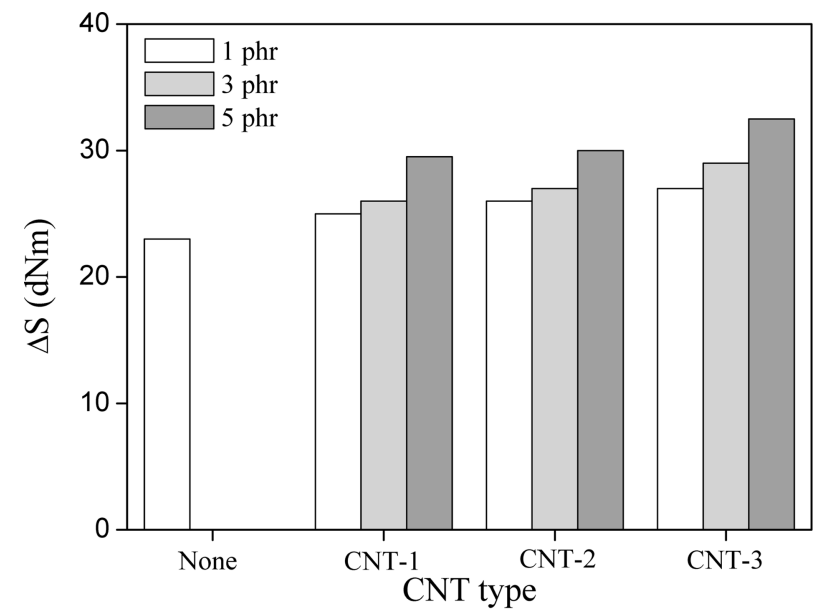

Fig. 5. Stress vs strain of CNTs. types; (a) CNT-1, (b) CNT-2 and (c) CNT-3.

addition of CNTs due to the absorption of curing agents on CNT surfaces in rubber matrix. This result was similar to the previous works [10,14]. However in case of the nanocomposites with CNT-1, the curing time decreased with the CNT content. It is probably because CNT-1 with better crystallinity and the thermal conductivity led to a fast crosslinking of rubbers in the composites.

Tensile property was measured by a tensile tester at a cross-head speed of $500 \mathrm{~mm} / \mathrm{min}$ and at room temperature, as described in ASTM D412. Strain-Stress curves of nanocomposites with three diameters of CNTs were shown in Fig. 5 and the corresponding mechanical properties were indicated in Table 3. Clearly there was the reinforcement as evidenced by considerable improvements in stiffness. In case of CNT-1 shown Fig. 5(a), with the addition of only 1 phr MWNTs, $120 \%$ increase in the tensile strength were achieved. The Stress slightly increased with the more loading of CNTs. In case of CNT-2 and CNT-3, relatively small increase in strength with 1 phr loading of CNTs was observed. But for the nanocomposites with 3 and $5 \mathrm{phr}$ CNTs, the improvement of strength was considerable. As shown in Table 4, 230\% increase in tensile strength was achieved with 5 phr of CNT-3. It could be thought that in case of CNT-1, even in the lower addition of CNTs the CNTs were easily entangled due to the higher aspect ratio of CNTs than those of other CNTs, which leads to the relatively small improvement in tensile strength for nanocomposites with 3 and 5 phr CNTs. In case of CNT-2 and CNT-3, even with a 5 phr addition of CNTs, there were smaller filler-filler interactions between CNTs compared to CNT-1.

The stresses at 100 and 300\% strain were shown in Table 4. The results were almost identical to the tensile strength. For $1 \mathrm{phr}$ loading of CNTs, the nanocomposite with CNT-1 showed the $70 \%$ and $80 \%$ increase in strength at $100 \%$ and $300 \%$ which are much larger than 20 and $10 \%$ increase observed for CNT-2 and -3 . Relatively small improvement 
Table 4. Mechanical properties of CNT-SBR nanocomposites

\begin{tabular}{cccccc}
\hline & $\begin{array}{c}\text { Contents } \\
\text { (phr) }\end{array}$ & $\begin{array}{c}\text { Stress at } \\
100 \% \\
(\mathrm{MPa})\end{array}$ & $\begin{array}{c}\text { Stress at } \\
300 \% \\
(\mathrm{MPa})\end{array}$ & $\begin{array}{c}\text { Stress at } \\
\text { break } \\
(\mathrm{MPa})\end{array}$ & $\begin{array}{c}\text { Strain at } \\
\text { break } \\
(\%)\end{array}$ \\
\hline None & 0 & 1.02 & 2.36 & 2.44 & 307 \\
\hline \multirow{3}{*}{ CNT-1 } & 1 & 1.70 & 4.36 & 5.36 & 333 \\
& 3 & 1.77 & 4.69 & 5.71 & 351 \\
& 5 & 1.60 & 4.60 & 6.37 & 382 \\
\hline \multirow{3}{*}{ CNT-2 } & 1 & 1.17 & 2.38 & 3.16 & 389 \\
& 3 & 1.55 & 3.72 & 5.32 & 401 \\
& 5 & 2.02 & 4.95 & 6.02 & 362 \\
\hline \multirow{3}{*}{ CNT-3 } & 1 & 1.22 & 2.61 & 3.90 & 401 \\
& 3 & 1.59 & 3.98 & 5.84 & 408 \\
& 5 & 1.89 & 5.08 & 8.05 & 423 \\
\hline
\end{tabular}
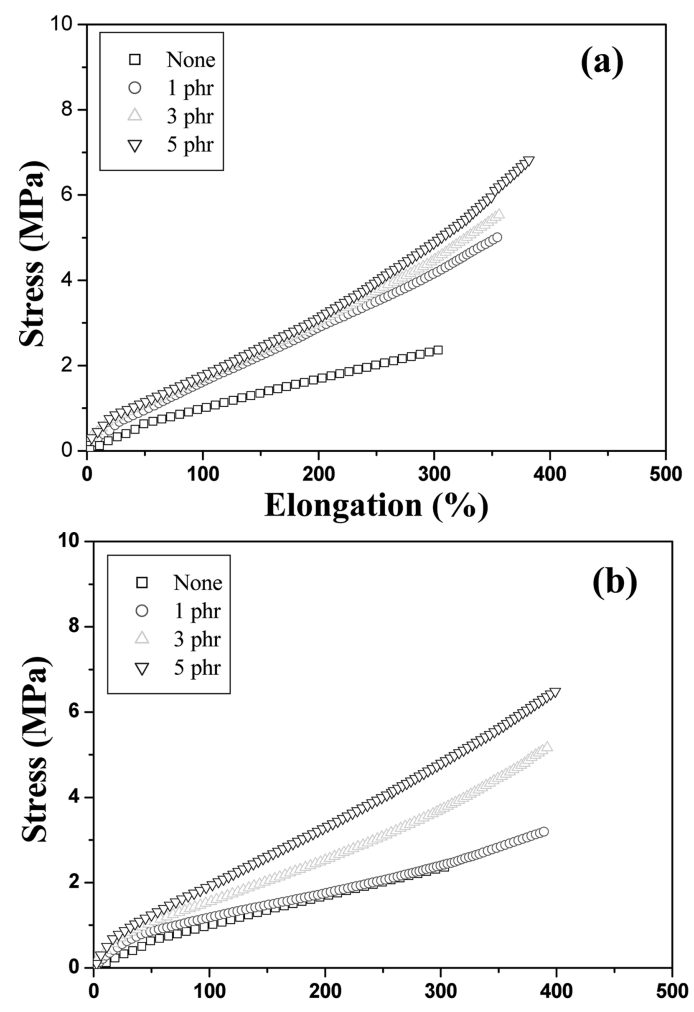

Elongation (\%)

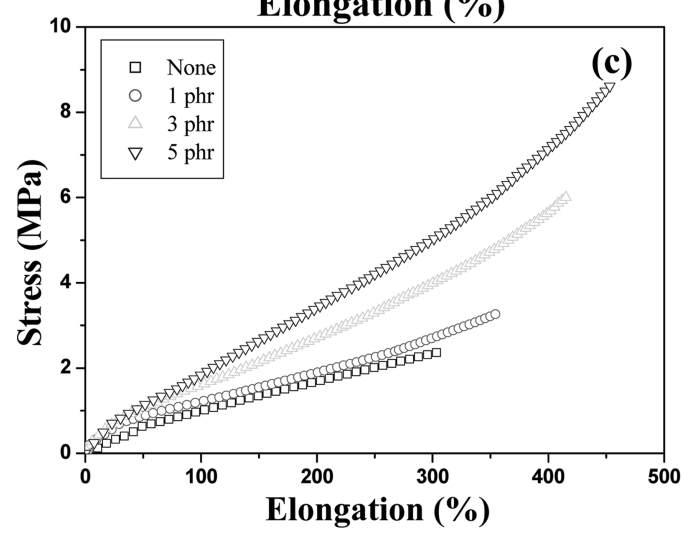

Fig. 6. Tensile strength vs the CNT contents and diameters. with increasing CNT content was also observed for at 100 and $300 \%$ strain.

\section{Conclusions}

In this study, we showed the effects of the diameter and content of CNTs on the physical properties of CNTs/SBR nanocomposites. For CNTs with a larger diameter (CNT-2 and CNT-3), the curing time, a minimum and maximum torque, and tensile strength of CNTs/SBR increased with the CNT content. For CNTs with a smaller diameter (CNT-1), curing time decreased with the CNT content. At the lowest CNT content, 1 phr, nanocomposites with a smaller diameter CNT-1 showed the highest tensile strength. However further increase of CNT-3 contents did improve the mechanical strength of nanocomposites probably due to the poor dispersion of CNTs within the rubber matrix.

\section{References}

[1] Seyhan, A.; Gojny, F.; Tanoglu, M.; Schulte, K. Euro. Polymer. J. 2007, 43, 374.

[2] Li, Y.; Shimizu, H. Polymer 2007, 48, 2203.

[3] Kanagaraj, S.; Varandaa, F.; Zhiltsova, T.; Oliveiraa, M.; Simoesa, J. Comp. Sci. Tech. 2007, 67, 3071.

[4] McIntosh, D.; Khabashesku, V.; Barrera, E. J. Phys. Chem. C, 2007, 111, 1592.

[5] Zhanga, C.; Nib, Q.; Fud, S.; Kurashikia, K. Comp. Sci. Tech. 2007, 67, 2973

[6] Treacy, M.; Ebbesen, T. W.; Gibson, J. M. Nature 1996, $381,678$.

[7] Dai, H.; Wong, E. W.; Lieber, C. M. Science 1996, 272, 523.

[8] Wong, E.; Sheehan, P.; Lieber, C. Science 1997, 277, 1971.

[9] Poncharal, P.; Wang, Z.; Ugarte, D.; Deheer, W. Science 1999, 283, 1513.

[10] Sui, G.; Zhong, W.; Yang, X.; Zhao, S. Macromol. Mater. Eng. 2007, 292, 1020.

[11] Sui, G.; Zhong, W. H.; Yang, X. P.; Yu, Y. H. Mater. Sci. Eng. A 2008, 485, 524.

[12] Kueseng, K.; Jacob, K. I. Eur. Polymer. J. 2007, 42, 220.

[13] Lopez, M. A.; Biagiotti, J.; Valentini, L.; Kenny, J. M. J. Appl. Polymer. Sci. 2006, 92, 3394.

[14] Zhong, W.; Li, J.; Lukehart, C.; Xu, L. Polymer. Nanocomposites. 2006, 26, 128.

[15] Bhattacharyyaa, S.; Sinturela, C.; Bahloula, O.; Saboungia, M.; Thomasb, S.; Salvetata, J. Carbon 2008, 46, 1037. 